\title{
VANISHING CONFIGURATIONS IN NETWORK DYNAMICS WITH ASYNCHRONOUS UPDATES
}

\author{
IAN H. DINWOODIE
}

(Communicated by David Levin)

\begin{abstract}
We consider Boolean dynamics for biological networks where stochasticity is introduced through asynchronous updates. An exact method is given for finding states which can reach a steady state with positive probability, and a method is given for finding states which cannot reach other steady states. These methods are based on computational commutative algebra. The algorithms are applied to dynamics of a cell survival network to determine node assignments that exclude termination in a cancerous state.
\end{abstract}

\section{INTRODUCTION}

In 14 Saadatpour et al. present a Boolean model of biological dynamics for cell cancer. In that work, one goal was to find configurations of the system which cannot lead to certain steady states related to cancer. In this paper we show how the questions and computations can be formulated exactly in terms of operations of computational commutative algebra. This is useful for three reasons: first, the uncertainty in simulation and random search is eliminated; second, the algebraic methods generalize easily to dynamics with more states than two; and third, existing commutative algebra software is highly developed so computations can be done simply by interfacing to existing software. We apply the methods to real and contrived examples. The algebraic approach will not scale to larger problems as well as simulation, so it will have limitations and thus we view it as complementary to other numerical methods.

Biological networks have been modeled as discrete dynamical systems for the purposes of understanding interactions and determining steady state solutions, and logical or Boolean models are the most tractable mathematically. Despite their simplicity, Boolean models have been used successfully for decades ([1], [9], 12], [17], [18], 19], 20]).

To account for uncertainty and variability in the timing of the interactions in the network, techniques for introducing randomness in the coordinate functions that define the dynamics have been of interest. These techniques are sometimes called "asynchronous" dynamics to distinguish them from the plain deterministic dynamics where the coordinate maps are simultaneously or synchronously applied to the present state, mapping it instantly to the next state. The asynchronous systems are Markovian and bring in probabilistic notions of the basin of attraction

Received by the editors June 25, 2012 and, in revised form, September 25, 2012.

2010 Mathematics Subject Classification. Primary 13P25, 62M86.

Key words and phrases. Asynchronous network, basin of attraction, Boolean network, Groebner basis, Markov chain. 
that extend the traditional definition. Here we will focus on two notions: a set of points that can reach a steady state with positive probability and a set of points that cannot reach any other steady state. We give algorithms for finding these sets and algorithms for low-dimensional conditions of inclusion and exclusion. We illustrate with examples of six and eleven nodes in Section 3.

\section{IDEALS OF BASINS OF ATTRACTION FOR ASYNCHRONOUS UPDATES}

In this section we show how to construct polynomials that vanish on a basin of attraction for randomized dynamics. The methods are related to [13, with the difference that the states of interest or "design points" here are not given explicitly. The points are studied after the ideal of polynomials is computed.

Consider a state space $\Omega:=\{0,1\}^{d}$, a $d$-fold product of logical or ON/OFF symbols 0 and 1 . These will be states or configurations on a network with $d$ nodes.

Let $F=\left(F_{1}, \ldots, F_{d}\right)$ be a transition map or transition function or update function on $\Omega$, where $F_{j}: \Omega \rightarrow\{0,1\}$ and $F: \Omega \rightarrow \Omega$. This map is deterministic; it is the simplified algebraic or logical model of interactions from one time step to the next and it is often called the "synchronous" update. Schemes to allow updates at different rates for different sites are called "asynchronous". Two common choices are "random node" (recommended by [14] and 22]), which are called "general asynchronous" or GA, and "random order" (implemented in [3]).

GA proceeds by choosing a node or coordinate $j \in\{1,2, \ldots, d\}$ with the uniform probability distribution, then changing the current state $\mathbf{s}=\left(s_{1}, \ldots, s_{j-1}, s_{j}\right.$, $\left.s_{j+1}, \ldots, s_{d}\right)$ to $\mathbf{s}^{\star}=\left(s_{1}, \ldots, s_{j-1}, F_{j}(\mathbf{s}), s_{j+1}, \ldots, s_{d}\right)$.

Random order chooses a permutation $\pi$ on the $d$ coordinates uniformly from the $d$ ! choices, then updates the states in that order to get a new state $\mathbf{s}^{\star}$ after going through intermediate updates $\mathbf{s}^{1}, \ldots, \mathbf{s}^{d-1}$ that are discarded after the computation:

$$
\begin{aligned}
& \mathbf{s}_{j}^{1}=\left\{\begin{array}{l}
\mathbf{s}_{j}, j \neq \pi(1), \\
F_{\pi(1)}(\mathbf{s}), j=\pi(1),
\end{array}\right. \\
& \mathbf{s}_{j}^{2}=\left\{\begin{array}{l}
\mathbf{s}_{j}^{1}, j \neq \pi(2), \\
F_{\pi(2)}\left(\mathbf{s}^{1}\right), j=\pi(2),
\end{array}\right. \\
& \mathbf{s}_{j}^{3}=\left\{\begin{array}{l}
\mathbf{s}_{j}^{2}, j \neq \pi(3), \\
F_{\pi(3)}\left(\mathbf{s}^{2}\right), j=\pi(3),
\end{array}\right. \\
& \quad \ldots \\
& \mathbf{s}_{j}^{\star}=\left\{\begin{array}{l}
\mathbf{s}_{j}^{d-1}, j \neq \pi(d), \\
F_{\pi(d)}\left(\mathbf{s}^{d-1}\right), j=\pi(d) .
\end{array}\right.
\end{aligned}
$$

Each of these random update methods gives a Markov chain $X_{0}, X_{1}, X_{2}, \ldots$ of sequences of states $\mathbf{s} \in \Omega$. Suppose the probability measures on trajectories (elements in $\Omega^{\infty}$ ) are denoted by $P^{G A}$ and $P^{\pi}$ respectively.

A steady state $\mathbf{p}=\left(p_{1}, \ldots, p_{d}\right) \in \Omega$ has the defining property that $F(\mathbf{p})=\mathbf{p}$; that is, it is fixed for the synchronous update. Then it follows that

$$
P^{G A}\left(X_{n}=\mathbf{p} \mid X_{0}=\mathbf{p}\right)=P^{\pi}\left(X_{n}=\mathbf{p} \mid X_{0}=\mathbf{p}\right)=1
$$

for all $n>0$. 
Define the set of points that may reach a steady state $\mathbf{p}$ :

$$
\begin{aligned}
& B_{i n, \mathbf{p}}^{G A}:=\bigcup_{n=0}^{\infty}\left\{\mathbf{s}: P^{G A}\left(X_{n}=\mathbf{p} \mid X_{0}=\mathbf{s}\right)>0\right\}, \\
& B_{i n, \mathbf{p}}^{\pi}:=\bigcup_{n=0}^{\infty}\left\{\mathbf{s}: P^{\pi}\left(X_{n}=\mathbf{p} \mid X_{0}=\mathbf{s}\right)>0\right\} .
\end{aligned}
$$

Each will be called an inclusive basin of attraction. Note that

$$
B_{i n, \mathbf{p}}^{\pi} \subset B_{i n, \mathbf{p}}^{G A}
$$

since if $\pi_{1}, \pi_{2}, \ldots, \pi_{n}$ is a sequence of permutations that connect a point $\mathbf{s} \in B_{i n, \mathbf{p}}^{\pi}$ to $\mathbf{p}$ in $P^{\pi}$ distribution, then the $n d$ single node updates that comprise the $n$ permutations give a positive probability of connecting $\mathbf{s} \rightarrow \mathbf{p}$ under the $P^{G A}$ distribution.

Our computational methods will primarily focus on $P^{G A}$, but conclusions will follow about $P^{\pi}$ based on certain inclusions, and these are summarized in Proposition 3.1.

A way to study $B_{i n, \mathbf{p}}^{G A}$ is through the set of polynomials that vanish on all points in $B_{i n, \mathbf{p}}^{G A}$, that is, its ideal:

$$
I\left(B_{i n, \mathbf{p}}^{G A}\right):=\left\{f(\mathbf{x}) \in \mathbb{C}[\mathbf{x}]: f\left(B_{i n, \mathbf{p}}^{G A}\right)=0\right\} .
$$

If $I$ is any ideal of polynomials, then define $\mathbf{V}(I):=\bigcap_{f \in I}\{\mathbf{s}: f(\mathbf{s})=0\}$, the simultaneous roots of all the equations in $I$. In particular, we can recover $B_{i n, \mathbf{p}}^{G A}$ :

$$
B_{i n, \mathbf{p}}^{G A}=\mathbf{V}\left(I\left(B_{i n, \mathbf{p}}^{G A}\right)\right) .
$$

It will be convenient for the algorithms of this section to use twice as many indeterminates as the number of coordinates $d$. Define the ring of polynomials $R=$ $\mathbb{C}\left[x_{1}, \ldots, x_{d}, y_{1}, \ldots, y_{d}\right]=\mathbb{C}[\mathbf{x}, \mathbf{y}]$. The algorithm below constructs the polynomials that vanish on $B_{i n, \mathbf{p}}^{G A}$ without numerically solving for the points in the set.

Define the ideals

$$
\begin{aligned}
I_{01} & =\left\langle x_{1}^{2}-x_{1}, \ldots, x_{d}^{2}-x_{d}, y_{1}^{2}-y_{1}, \ldots, y_{d}^{2}-y_{d}\right\rangle, \\
F_{j x y} & =\left\langle x_{1}-y_{1}, \ldots, x_{j-1}-y_{j-1}, F_{j}(\mathbf{x})-y_{j}, x_{j+1}-y_{j+1}, \ldots, x_{d}-y_{d}\right\rangle, \\
F_{j y x} & =\left\langle y_{1}-x_{1}, \ldots, y_{j-1}-x_{j-1}, F_{j}(\mathbf{y})-x_{j}, y_{j+1}-x_{j+1}, \ldots, y_{d}-x_{d}\right\rangle, \\
I_{y p} & =\left\langle y_{1}-p_{1}, \ldots, y_{d}-p_{d}\right\rangle .
\end{aligned}
$$

Define the ideal $I_{1}$ by

$$
I_{1}=\left(\bigcap_{j=1}^{d} F_{j x y}+I_{y p}+I_{01}\right) \cap \mathbb{C}[\mathbf{x}] .
$$

Now define recursively a sequence of ideals $I_{2}, I_{3}, I_{4}, \ldots$ by

$$
\begin{aligned}
J & =\left(\bigcap_{j=1}^{d} F_{j y x}+I_{i}+I_{01}\right) \cap \mathbb{C}[\mathbf{y}], \\
I_{i+1} & =\left(\bigcap_{j=1}^{d} F_{j x y}+J+I_{01}\right) \cap \mathbb{C}[\mathbf{x}], \quad i=1,2,3, \ldots
\end{aligned}
$$


Theorem 2.1 says to stop the iteration when $\operatorname{dim} R /\left(I_{i}+I_{01}\right)$ repeats in order to get the polynomials that vanish on the basin of attraction $B_{i n, \mathbf{p}}^{G A}$. The method above is a variation on the simpler method for synchronous updates in [6].

Theorem 2.1. Let $F(\mathbf{p})=\mathbf{p}$. There exists $i^{\star}<\infty$ such that $\operatorname{dim} R /\left(I_{i^{\star}}+I_{01}\right)=$ $\operatorname{dim} R /\left(I_{i^{\star}+1}+I_{01}\right)$, and for such an integer

$$
I\left(B_{i n, \mathbf{p}}^{G A}\right)=I_{i^{\star}}
$$

as an ideal within $\mathbb{C}[\mathbf{x}]$.

Proof. Write the set $B_{i n, \mathbf{p}}^{G A}=\bigcup_{i=0}^{\infty}\left\{\mathbf{s} \in \Omega: P^{G A}\left(X_{i}=\mathbf{p} \mid X_{0}=\mathbf{s}\right)>0\right\}$. Note that the sets in this union $\left\{\mathbf{s} \in \Omega: P^{G A}\left(X_{i}=\mathbf{p} \mid X_{0}=\mathbf{s}\right)>0\right\}$ are increasing by inclusion, since $\mathbf{p}$ is a steady state. Since $\Omega$ is finite, there is an index $i^{\star}$ when

$$
\left\{\mathbf{s} \in \Omega: P^{G A}\left(X_{i^{\star}}=\mathbf{p} \mid X_{0}=\mathbf{s}\right)>0\right\}=\left\{\mathbf{s} \in \Omega: P^{G A}\left(X_{i^{\star}+1}=\mathbf{p} \mid X_{0}=\mathbf{s}\right)>0\right\},
$$

and then these sets remain the same for $i \geq i^{\star}$, as follows. If $\mathbf{s}$ satisfies $P^{G A}\left(X_{i^{\star}+2}\right.$ $\left.=\mathbf{p} \mid X_{0}=\mathbf{s}\right)>0$, then by the Markov property

$$
\begin{aligned}
0 & <\sum_{\mathbf{t}} P^{G A}\left(X_{i^{\star}+1}=\mathbf{p} \mid X_{0}=\mathbf{t}\right) P^{G A}\left(X_{1}=\mathbf{t} \mid X_{0}=\mathbf{s}\right) \\
& \leq C \sum_{\mathbf{t}} P^{G A}\left(X_{i^{\star}}=\mathbf{p} \mid X_{0}=\mathbf{t}\right) P^{G A}\left(X_{1}=\mathbf{t} \mid X_{0}=\mathbf{s}\right) \\
& =C P^{G A}\left(X_{i^{\star}+1}=\mathbf{p} \mid X_{0}=\mathbf{s}\right)
\end{aligned}
$$

for some constant $C>0$, using the assumption that the two integrands indexed by $i^{\star}$ and $i^{\star}+1$ are positive at the same values of the argument $\mathbf{t}$. This shows that $\mathbf{s}$ also has a positive probability of reaching $\mathbf{p}$ at time $i^{\star}+1$, and this will be the case for all times $i>i^{\star}$.

Let $B_{i}:=\left\{\mathbf{s} \in \Omega: P^{G A}\left(X_{i}=\mathbf{p} \mid X_{0}=\mathbf{s}\right)>0\right\}$, let $I_{y, i}$ be the polynomials in indeterminates $y_{1}, \ldots, y_{d}$ that vanish on $B_{i}$, and let $I_{x, i}$ be the same polynomials in $x_{1}, \ldots, x_{d}$. Now for convenience let $f_{j}(\mathbf{s})=\left(s_{1}, s_{2}, \ldots, s_{j-1}, F_{j}(\mathbf{s}), s_{j+1}, \ldots, s_{d}\right)$ and write

$$
\begin{aligned}
B_{i} & =\bigcup_{j=1}^{d}\left\{\mathbf{s} \in \Omega: P^{G A}\left(X_{i-1}=\mathbf{p} \mid X_{0}=f_{j}(\mathbf{s})\right)>0\right\} \\
& =\bigcup_{j=1}^{d}\left\{\mathbf{s} \in \Omega: \mathbf{t} \in B_{i}, \mathbf{t}=f_{j}(\mathbf{s})\right\} \\
& =\left\{\mathbf{s}:(\mathbf{s}, \mathbf{t}) \in \bigcup_{j=1}^{d} \mathbf{V}\left(F_{j x y}+I_{y, i-1}+I_{01}\right)\right\} \\
& =\left\{\mathbf{s}: \mathbf{s} \in \mathbf{V}\left(\bigcap_{j=1}^{d}\left(F_{j x y}+I_{y, i-1}+I_{01}\right) \cap \mathbb{C}[\mathbf{x}]\right)\right\},
\end{aligned}
$$

where we have applied the Extension Theorem ([4, p. 25) to the ideal $\bigcap_{j=1}^{d} F_{j x y}+$ $I_{y, i-1}+I_{01}$ - the univariate polynomials in $I_{01}$ make all partial solutions in the elimination ideal extend to complete solutions. 
Now continuing the recursive formula back to $i=0$, we have $I_{y, 0}=I_{y p}$. Thus we have the construction

$$
I_{x, i}=\left(\bigcap_{j=1}^{d} F_{j x y}+I_{y, i-1}+I_{01}\right) \cap \mathbb{C}[\mathbf{x}],
$$

which is more easily implemented by alternating $\mathbf{x}$ and $\mathbf{y}$ variable elimination. Note then that the index $i$ in the statement of the theorem is the odd index $2 i-1$ in the proof.

Finally, since $I_{01}$ contains the univariate polynomials $x_{j}^{2}-x_{j}, y_{j}^{2}-y_{j}$, the ideal $I_{x, i}+I_{01}$ is radical and 0-dimensional (Seidenberg's Lemma [10]), so its size can be measured as the dimension of the quotient vector space $R /\left(I_{x, i}+I_{01}\right)$. When the dimensions repeat, the sets are the same and the iteration stops.

It follows from the above result and the ideal-variety correspondence ([4, p. 21) that we can get the points $B_{i n, \mathbf{p}}^{G A}$ by solving the equations that generate the ideal $I\left(B_{i n, \mathbf{p}}^{G A}\right)$.

Note that $I_{i^{\star}}$ is 0 -dimensional in $\mathbb{C}[\mathbf{x}]$ but not in $\mathbb{C}[\mathbf{x}, \mathbf{y}]$. Since it contains each $x_{j}^{2}-x_{j}$, the formula for counting holds:

$$
\left|B_{i n, \mathbf{p}}^{G A}\right|=\operatorname{dim} \frac{\mathbb{C}[\mathbf{x}, \mathbf{y}]}{I_{i^{\star}}+I_{y p}}=2^{-d} \operatorname{dim} \frac{\mathbb{C}[\mathbf{x}, \mathbf{y}]}{I_{i^{\star}}+I_{01}} .
$$

Define the set of points that cannot leave the inclusive basin of attraction of state $\mathbf{p}$ :

$$
B_{e x, \mathbf{p}}^{G A}:=\bigcap_{n=0}^{\infty}\left\{\mathbf{s}: P^{G A}\left(X_{n} \notin B_{i n, \mathbf{p}}^{G A} \mid X_{0}=\mathbf{s}\right)=0\right\} .
$$

This will be called the exclusive basin of attraction. Observe that

$$
\begin{aligned}
\mathbf{p} & \in B_{e x, \mathbf{p}}^{G A}, \\
B_{e x, \mathbf{p}}^{G A} & \subset B_{i n, \mathbf{p}}^{G A} .
\end{aligned}
$$

Also, the exclusive basins are disjoint, as follows.

Proposition 2.1. $B_{e x, \mathbf{p}}^{G A} \cap B_{e x, \mathbf{q}}^{G A}=\emptyset$ and $\mathbf{q} \notin B_{i n, \mathbf{p}}^{G A}$ if $\mathbf{p}$ and $\mathbf{q}$ are different steady states.

Proof. Suppose $\mathbf{s} \in B_{e x, \mathbf{p}}^{G A} \cap B_{e x, \mathbf{q}}^{G A}$. Then $\mathbf{s} \in B_{i n, \mathbf{q}}^{G A}$ so there would be a time step $n$ when $P^{G A}\left(X_{n}=\mathbf{q} \mid X_{0}=\mathbf{s}\right)>0$. But $\mathbf{q} \notin B_{i n, \mathbf{p}}^{G A}$, since $\mathbf{q}$ is a steady state for $F$, so the Markov chain $P^{G A}$ started at $\mathbf{q}$ can never move. Thus we have $P^{G A}\left(X_{n} \notin B_{i n, \mathbf{p}}^{G A} \mid X_{0}=\mathbf{s}\right)>0$, a contradiction to the definition of $B_{e x, \mathbf{p}}^{G A}$.

Now we describe a method to find $B_{e x, \mathbf{p}}^{G A}$ which will be used in Section 3. Intuitively the method finds sets of points that enter the complement of $B_{i n, \mathbf{p}}^{G A}$ in 1 step, 2 steps, 3 steps, $\ldots$ of the Markov chain $P^{G A}$, and at the end removes these points from $B_{i n, \mathbf{p}}^{G A}$ algebraically with the colon operation.

Let $I_{x}\left(B_{i n, \mathbf{p}}^{G A}\right)$ be the ideal of polynomials that vanish on $B_{i n, \mathbf{p}}^{G A}$ in terms of indeterminates $x_{1}, \ldots, x_{d}$, and let $I_{y}\left(B_{i n, \mathbf{p}}^{G A}\right)$ be the same polynomials using $y_{1}, \ldots, y_{d}$. 
Define

$$
\begin{aligned}
F_{0 x y}=F_{0 y x} & =\left\langle x_{1}-y_{1}, \ldots, x_{d}-y_{d}\right\rangle \\
E_{1} & =\left(\bigcap_{j=0}^{d} F_{j x y}+I_{01}: I_{y}\left(B_{i n, \mathbf{p}}^{G A}\right)\right) \cap \mathbb{C}[\mathbf{x}]
\end{aligned}
$$

and then define recursively

$$
\begin{aligned}
J & =\left(\bigcap_{j=0}^{d} F_{j y x}+E_{i}+I_{01}\right) \cap \mathbb{C}[\mathbf{y}], \\
E_{i+1} & =\left(\bigcap_{j=0}^{d} F_{j x y}+J+I_{01}\right) \cap \mathbb{C}[\mathbf{x}], i=1,2,3, \ldots
\end{aligned}
$$

Theorem 2.2. There exists $i^{\star}$ such that $E_{i^{\star}+1}=E_{i^{\star}}$, and then

$$
I\left(B_{e x, \mathbf{p}}^{G A}\right)=\left(I_{01}: E_{i^{\star}}\right) \cap \mathbb{C}[\mathbf{x}]
$$

as an ideal in $\mathbb{C}[\mathbf{x}]$.

Proof. Define a new Markov chain $P^{G A, 0}$ to be like the GA process but where each coordinate is chosen for update with probability $\frac{1}{d+1}$, and the possibility to hold at the current state has probability $\frac{1}{d+1}$ as well. Then

$$
\begin{aligned}
& \bigcup_{n=0}^{i}\left\{\mathbf{s}: P^{G A}\left(X_{n} \notin B_{i n, \mathbf{p}}^{G A} \mid X_{0}=\mathbf{s}\right)>0\right\} \\
& \quad=\left\{\mathbf{s}: P^{G A, 0}\left(X_{i} \notin B_{i n, \mathbf{p}}^{G A} \mid X_{0}=\mathbf{s}\right)>0\right\},
\end{aligned}
$$

and these sets are increasing in the index $i$ by inclusion. Then the proof closely follows the proof of Theorem 2.1 with $I_{y p}$ replaced by $I_{01}: I_{y}\left(B_{i n, \mathbf{p}}^{G A}\right)$. We get

$$
E_{i^{\star}}=I\left(\bigcup_{n=0}^{\infty}\left\{\mathbf{s} \in \Omega: P^{G A}\left(X_{n} \notin B_{i n, \mathbf{p}}^{G A} \mid X_{0}=\mathbf{s}\right)>0\right\}\right)=I\left(\bar{B}_{e x, \mathbf{p}}^{G A}\right)
$$

in $\mathbb{C}[\mathbf{x}]$, and so

$$
\mathbf{V}\left(I_{01}: E_{i^{\star}} \cap \mathbb{C}[\mathbf{x}]\right)=\left\{\mathbf{s} \in \Omega:(\mathbf{s}, \mathbf{t}) \in\left(\Omega \backslash \bar{B}_{e x, \mathbf{p}}^{G A}\right) \times \Omega\right\}=B_{e x, \mathbf{p}}^{G A}
$$

Now we consider a contrived example. We will use a simplified notation:

$$
\begin{aligned}
& I_{i n, \mathbf{p}}^{G A}=I\left(B_{i n, \mathbf{p}}^{G A}\right), \\
& I_{e x, \mathbf{p}}^{G A}=I\left(B_{e x, \mathbf{p}}^{G A}\right) .
\end{aligned}
$$

Example 2.1. For illustration consider a map $F$ on binary triples described by

$$
001 \rightarrow 010 \rightarrow 011 \rightarrow 111
$$

with all other points including 111 fixed for $F$. The map $F$ can be written with polynomial coordinates $\left(F_{1}, F_{2}, F_{3}\right)$, where for example

$$
\begin{aligned}
& F_{1}(\mathbf{x})=-x_{1} x_{2} x_{3}+x_{2} x_{3}+x_{1}, \\
& F_{2}(\mathbf{x})=x_{1} x_{2} x_{3}-x_{1} x_{3}-x_{2} x_{3}+x_{2}+x_{3}, \\
& F_{3}(\mathbf{x})=-x_{1} x_{2} x_{3}+x_{1} x_{3}+x_{2} x_{3}
\end{aligned}
$$

on $\Omega$. 
Then $B_{i n, \mathbf{1}}^{G A}=B_{i n, \mathbf{1}}^{\pi}=\{001,010,011,111\}$ but $B_{e x, \mathbf{1}}^{G A}=\{010,011,111\}$ and $001 \epsilon$ $B_{i n, \mathbf{1}}^{G A} \cap B_{i n, \mathbf{0}}^{G A}$. The state 001 can reach 111 in the GA scheme by coordinate choices 2 , then 1 , and it can also reach steady state 000 by a single choice of coordinate 3. The standard Gröbner basis for $I_{i n, 1}^{G A}$ with graded reverse lex order is given by Singular [7] as $\left\{x_{3}^{2}-x_{3}, x_{2} x_{3}-x_{2}-x_{3}+1, x_{1} x_{3}-x_{1}, x_{2}^{2}-x_{2}, x_{1} x_{2}-x_{1}, x_{1}^{2}-x_{1}\right\}$.

\section{VANishing CONFIGURATIONS}

In this section we are concerned with answering questions about the nature of points in and out of the basins of attraction, in order to determine simple conditions for inclusion and exclusion. Here the polynomials will be $\mathbb{C}[\mathbf{x}]$, so the ideals from Section 2 will be mapped over the obvious way $\left(x_{j} \rightarrow x_{j}, y_{j} \rightarrow 0\right)$. Now $I_{i n, \mathbf{p}}^{G A}$ and $I_{e x, \mathbf{p}}^{G A}$ are ideals of polynomials in $x_{1}, \ldots, x_{d}$, and $I_{01}=\left\langle x_{j}^{2}-x_{j}, j=1, \ldots, d\right\rangle$.

Theorem 2.1 gives a way to find states that are not in an undesirable basin of attraction: solve the equations in $I_{01}: I_{i n, \mathbf{p}}^{G A}$. However, for applications it is useful to have low-dimensional characterizations of exclusion. Are there values at one or two coordinates that imply exclusion? The sets of nodes and particular values for these nodes that guarantee exclusion from a basin of attraction will be called vanishing configurations. These would be interpreted as nodes where values could be fixed to force exclusion. We will also treat the question of inclusion, where the goal is to make sure a state belongs to a desirable basin of attraction.

The following proposition summarizes conclusions about the meaning of a state $\mathbf{s}$ not being in an inclusive basin of attraction and of being in an exclusive basin of attraction. For motivation, $\mathbf{p}$ may be an undesirable steady state, and we will want to find conditons on states that prevent them from reaching $\mathbf{p}$.

Proposition 3.1. The quotient ideal $I_{01}: I_{\text {in, }}^{G A}$ has variety $\mathbf{V}\left(I_{01}: I_{i n, \mathbf{p}}^{G A}\right)=$ $\left\{\mathbf{s} \in \Omega: \mathbf{s} \notin B_{i n, \mathbf{p}}^{G A}\right\}$. If $\mathbf{s} \notin B_{i n, \mathbf{p}}^{G A}$, then $\mathbf{s}$ cannot reach $\mathbf{p}$ under $P^{G A}$ nor $P^{\pi}$. Also, if $\mathbf{s} \in B_{e x, \mathbf{q}}^{G A}$, then $\mathbf{s}$ cannot reach a steady state $\mathbf{p} \neq \mathbf{q}$ under $P^{G A}$ nor $P^{\pi}$.

Proof. The ideal $I_{01}$ is radical, so $\mathbf{V}\left(I_{01}: I_{i n, \mathbf{p}}^{G A}\right)$ is the smallest variety containing the difference $\Omega \backslash B_{i n, \mathbf{p}}^{G A}$ (还, p. 23). But this set difference is a variety, so the two are equal.

If $\mathbf{s} \notin B_{i n, \mathbf{p}}^{G A}$, then $\mathbf{s}$ cannot reach $\mathbf{p}$ under $P^{G A}$ by definition; then nor by $P^{\pi}$ by (3). Clearly $\mathbf{s} \in B_{e x, \mathbf{q}}^{G A}$ cannot reach $\mathbf{p}$ under $P^{G A}$ because $\mathbf{p} \notin B_{i n, \mathbf{q}}^{G A}$. If $\mathbf{s}$ could reach $\mathbf{p}$ under $P^{\pi}$, then it could do so under $P^{G A}$ as well, so this is impossible.

Proposition 3.2. For $b \in\{0,1\}$, the condition

$$
\left\langle x_{j}-b\right\rangle+I_{i n, \mathbf{p}}^{G A}=\langle 1\rangle
$$

implies that every state $\mathbf{s} \in \Omega$ with $s_{j}=b$ does not belong to $B_{i n, \mathbf{p}}^{G A}$.

Proof. If there were $\mathbf{s} \in B_{i n, \mathbf{p}}^{G A}$ with $s_{j}=b$, then all the polynomials in $I_{i n, \mathbf{p}}^{G A}$ would vanish on $\mathbf{s}$, as well as all of them in $\left\langle x_{j}-b\right\rangle$ (being multiples of the generator $\left.x_{j}-b\right)$, as well as all of them in $I_{01}$. Thus $\mathbf{s} \in \mathbf{V}\left(\left\langle x_{j}-b\right\rangle+I_{i n, \mathbf{p}}^{G A}+I_{01}\right)$, but there could be no solutions if this ideal were $\langle 1\rangle=\mathbb{C}[\mathbf{x}]$.

Finding a $b$ to satisfy the above algebraic condition would involve a search and computation over $2 d$ node-value pairs. If one is searching only for nodes where $b=0$, this would correspond intuitively to a situation where the steady state $\mathbf{p}$ characterizes a disease condition and the value at node $j$ satisfies $s_{j}=1$ for all 
states in the inclusive basin of attraction; that is, node $j$ is "stuck-at-ON" (see 14] for terminology). Then setting $s_{j}=0$ would guarantee exclusion from the basin of attraction of the disease condition. To find joint states guaranteeing exclusion, the algebraic condition

$$
\left\langle 1-\left(1-b_{1}-x_{i}\right) \cdot\left(1-b_{2}-x_{j}\right)\right\rangle+I_{i n, \mathbf{p}}^{G A}=\langle 1\rangle
$$

implies that any state $\mathbf{s}$ with $\left(s_{i}, s_{j}\right)=\left(b_{1}, b_{2}\right)$ is not in $B_{i n, \mathbf{p}}^{G A}$; here the search is over $4 \cdot\left(\begin{array}{l}d \\ 2\end{array}\right)$ pairs and values. We also mention that another tool for finding vanishing configurations is the Monte Carlo sampling on $B_{i n, \mathbf{p}}^{G A}$, and then randomly sampled states $\mathbf{s}$ can be studied with pairwise scatterplots over all coordinate choices $(i, j)$ in $1, \ldots, d$. Pairs of states $\left(b_{1}, b_{2}\right)$ that do not show up are likely vanishing configurations if the sampling is done well. The sampling may be difficult; see [5] for methods based on sequential importance sampling. Also, a universal Gröbner basis [2] would be useful, as all the elimination ideals would be available simultaneously, and this would make it possible to find variables and particular values that cannot solve the equations. However, computational methods are not widely implemented.

Insight into how many nodes need to be considered jointly for a vanishing configuration can be obtained with the Hilbert function for the ideal $I_{i n, \mathbf{p}}^{G A}$ as we see in Theorem 3.1 below. A vanishing configuration of size $k$ for an inclusive basin of attraction $B_{i n, \mathbf{p}}^{G A}$ is a $k$-tuple of indices $\mathbf{i}=\left(i_{1}, \ldots, i_{k}\right)\left(1 \leq i_{1}<i_{2}<\cdots<i_{k} \leq d\right)$ and binary values $b_{1}, \ldots, b_{k}$ such that

$$
\left\{\mathbf{s} \in \Omega: \mathbf{s}_{\mathbf{i}}=\left(b_{1}, \ldots, b_{k}\right)\right\} \cap B_{i n, \mathbf{p}}^{G A}=\emptyset,
$$

or, in other words, a state $\mathbf{s}$ with binary values $b_{1}, \ldots, b_{k}$ at sites $\mathbf{i}$ does not belong to $B_{i n, \mathbf{p}}^{G A}$. The condition analogous to (12) would involve $2^{k}\left(\begin{array}{l}d \\ k\end{array}\right)$ possible configurations to examine.

The Hilbert function is easy to compute compared to searching over size $k=$ $1,2,3, \ldots$ together with the corresponding $2^{k}$ states to satisfy condition (12). The affine Hilbert function for the ideal $I$ is the function $H_{I}(t), t=0,1,2, \ldots$, given by

$$
H_{I}(t)=\operatorname{dim} \mathbb{C}[\mathbf{x}]_{\leq t} / I_{\leq t}, t=0,1,2, \ldots,
$$

where $\mathbb{C}[\mathbf{x}]_{<t}$ is the vector space of polynomials of total degree at most $t$ and similarly for $I_{\leq t}$.

Theorem 3.1. Let $I=I_{\text {in, }}^{G A}$, and let $t^{\star}$ be the first positive integer where $H_{I}\left(t^{\star}\right)<$ $\sum_{i=0}^{t^{\star}}\left(\begin{array}{c}d \\ i\end{array}\right)$, if one exists. Then there is no vanishing configuration of size $k<t^{\star}$.

Proof. The Hilbert function for $I_{01}$ is given by $H_{01}(t)=\sum_{i=0}^{t}\left(\begin{array}{c}d \\ i\end{array}\right)$, because the dimension of $\mathbb{C}[\mathbf{x}]_{\leq t}=\left(\begin{array}{c}d+t \\ t\end{array}\right)$ and the dimension of $I_{01, \leq t}$ is the number of integer solutions to $n_{0}+n_{1}+\ldots+n_{d}=t$ with one of the $n_{i}$ at least 2 , for inclusion in the ideal $I_{01}$. These solutions number $\left(\begin{array}{c}d+t \\ t\end{array}\right)-\sum_{i=0}^{t}\left(\begin{array}{c}d \\ i\end{array}\right)$, the total number of solutions minus the number with all values $n_{j} \leq 1$. Now assume $H_{I}(t)=H_{01}(t), t<t^{\star}$, and suppose there is a vanishing configuration of size $k<t^{\star}$. Then $I_{\leq t}$ would be spanned by $I_{01, \leq t}$ as vector spaces over $\mathbb{C}$ since $I_{01, \leq t} \subset I_{\leq t}$ and they have the same dimension, and in particular every polynomial in $I_{\leq t}$ is in the ideal $I_{01}$. If there were a vanishing configuration of size $k<t^{\star}$, then there would be a squarefree polynomial of total degree at most $k$ that vanishes on $B_{i n, \mathbf{p}}^{G A}$, namely $p(\mathbf{x})=$ $\prod_{j=1}^{k}\left(1-b_{j}-x_{i_{j}}\right)$ for vanishing configuration $\left(\mathbf{i},\left(b_{1}, \ldots, b_{k}\right)\right)$, and then $p \in I_{\leq k}$. 
But no square-free polynomial can be in the ideal $I_{01}$, by the division algorithm, which contradicts the existence of the vanishing configuration.

While we have just treated ways to find configurations that guarantee exclusion from a basin of attraction, one may also be interested in characterizing states in a way that guarantees inclusion in the exclusive basin of attraction. These should be called sufficient configurations. The idea is related to identifying favorable "stuckat-ON/OFF" configurations. Here we have the following analog of Proposition 3.2.

Proposition 3.3. For $b \in\{0,1\}$, the condition

$$
\left\langle x_{j}-b\right\rangle+I_{01}: I_{e x, \mathbf{p}}^{G A}=\langle 1\rangle
$$

implies that every state $\mathbf{s} \in \Omega$ with $s_{j}=b$ does belong to $B_{e x, \mathbf{p}}^{G A}$.

Proof. The condition implies that there can be no binary solutions s with $s_{j}=b$ to the equations that vanish on $\Omega \backslash \bar{B}_{e x, \mathbf{p}}^{G A}$.

The condition for joint states $\left(b_{1}, b_{2}\right)$ such that $\mathbf{s}_{(i, j)}=\left(b_{1}, b_{2}\right)$ implies membership in $B_{e x, \mathbf{p}}^{G A}$ is

$$
\left\langle 1-\left(1-b_{1}-x_{i}\right) \cdot\left(1-b_{2}-x_{j}\right)\right\rangle+I_{01}: I_{e x, \mathbf{p}}^{G A}=\langle 1\rangle .
$$

The application would be where $\mathbf{p}$ is a steady state related to some favorable condition, and the property $\mathbf{s}_{\mathbf{i}}=\left(b_{1}, b_{2}\right)$ guarantees membership in the basin of attraction that can only lead to steady state $\mathbf{p}$.

In our examples, all computations were done with Singular [7, including its elim.lib library for variable elimination. Hilbert functions were computed with hilb, and counting 0-dimensional sets were done with vdim.

Example 3.1. In Example 2.1, one sees that the smallest vanishing configuration in $B_{i n, \mathbf{1}}^{G A}$ is of size $k=2$ and involves indices $\mathbf{i}=(1,2)$ and values $\left(b_{1}, b_{2}\right)=(1,0)$. One can check condition (11) quickly. The Hilbert function drops down from $\sum_{i=0}^{t}\left(\begin{array}{l}3 \\ i\end{array}\right)$ at $t^{\star}=2$, consistent with Theorem 3.1. (For $B_{e x, \mathbf{1}}^{G A}, b=0$ in the second coordinate is vanishing and Proposition 3.2 can be applied to $I_{e x, \mathbf{1}}^{G A}$.) Furthermore, $\mathbf{i}=(1,2)$ and $\left(b_{1}, b_{2}\right)=(0,1)$ is a sufficient configuration for inclusion in $B_{e x, \mathbf{1}}^{G A}$, and condition (14) applies.

Example 3.2. Here we consider an example from [14. An update $x_{1}$ or $x_{2}$ is written as a polynomial in the form $x_{1}+x_{2}-x_{1} \cdot x_{2}$, and $x_{1}$ and $x_{2}$ is written $x_{1} \cdot x_{2}$. The polynomial defined at $(1)$ is $f_{K}(x)=x^{2}+x$. In the table below we describe a map $F$ on $d=6$ dimensions taken from Table 1 of [14, where the "update" indicates the next time step.

\begin{tabular}{|l|c|c|}
\hline node & update & indeterminate \\
\hline S1P & S1P $^{*}=$ NOT (Ceramide OR Apoptosis) & $x_{1}$ \\
FLIP & FLIP* NOT (DISC OR Apoptosis) $_{2}$ & $x_{2}$ \\
Fas & Fas $^{*}=$ NOT (S1P OR Apoptosis) & $x_{3}$ \\
Ceramide & Ceramide* Fas AND NOT (S1P OR Apoptosis) $^{*}$ & $x_{4}$ \\
DISC & DISC* $=$ (Ceramide OR (Fas AND NOT FLIP)) & $x_{5}$ \\
& AND NOT Apoptosis & $x_{6}$ \\
Apoptosis & Apoptosis* DISC OR Apoptosis & \\
\hline
\end{tabular}


This dynamic model has two steady states: a disease steady state $\mathbf{p}_{D}=1100000$,

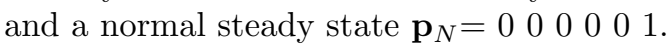

Calculations in Singular based on Theorems 2.1 and 2.2 show that the sizes of the inclusive basins of attraction are 61 and 28 for normal and disease respectively. The exclusive basins of attraction have sizes 36 and 3 , and a Gröbner basis for $I_{e x, \mathbf{p}_{D}}^{G A}$ with graded reverse lex order is given by $\left\{x_{6}, x_{5}, x_{4}, x_{1}-1, x_{3}^{2}-x_{3}, x_{2} x_{3}-x_{3}, x_{2}^{2}-x_{2}\right\}$.

The intersection of the inclusive basins of attraction counts 25 points (this is computed with vdim for the vector space dimension of the quotient $\mathbb{C}[\mathbf{x}] /$ $\left.\left(I_{i n, \mathbf{p}_{N}}^{G A}+I_{i n, \mathbf{p}_{D}}^{G A}\right)\right)$. These numbers agree with Figure 4 of [14].

The Hilbert function on the ideal $I\left(B_{i n, \mathbf{p}_{D}}^{G A}\right)$ shows $t^{\star}=1$, and a vanishing configuration is found to be $s_{6}=1$ using Proposition 3.2. Thus any state $\mathbf{s}$ with $s_{6}=1$ cannot converge to the disease steady state $\mathbf{p}_{D}$. An application of Proposition 3.3 shows that this condition also forces $\mathbf{s}$ to be in the exclusive basin of attraction for the normal state $B_{e x, \mathbf{p}_{N}}^{G A}$. These conclusions are consistent with Figure 4 of [14].

Example 3.3. Consider an 11-node T cell signalling model relevant to the network of [15. The signalling logical model described below has four steady states:

$$
\begin{array}{lllllllllll}
0 & 0 & 0 & 0 & 0 & 0 & 0 & 0 & 0 & 0 & 0 \\
1 & 1 & 1 & 1 & 0 & 1 & 1 & 1 & 1 & 1 & 1 \\
1 & 1 & 0 & 1 & 1 & 1 & 1 & 1 & 1 & 1 & 1 \\
1 & 1 & 1 & 1 & 1 & 1 & 1 & 1 & 1 & 1 & 1 .
\end{array} .
$$

The dynamics for this model are defined precisely by

\begin{tabular}{|l|c|c|}
\hline node & logical update & polynomial \\
\hline raf $=x_{1}$ & PKA or PKC & $x_{8}+x_{9}-x_{8} x_{9}$ \\
mek $=x_{2}$ & raf or PKA or PKC & $1-\left(1-x_{1}\right)\left(1-x_{8}\right)\left(1-x_{9}\right)$ \\
plcg $=x_{3}$ & plcg & $x_{3}$ \\
PIP $2=x_{4}$ & plcg or PIP3 & $x_{3}+x_{5}-x_{3} x_{5}$ \\
PIP3 $=x_{5}$ & PIP3 & $x_{5}$ \\
erk $=x_{6}$ & mek or PKA & $x_{2}+x_{8}-x_{2} x_{8}$ \\
akts $=x_{7}$ & PIP3 or erk or PKA & $1-\left(1-x_{8}\right)\left(1-x_{6}\right)\left(1-x_{5}\right)$ \\
PKA $=x_{8}$ & PKC & $x_{9}$ \\
PKC $=x_{9}$ & plcg or PIP2 & $x_{3}+x_{4}-x_{3} x_{4}$ \\
P38 $=x_{10}$ & PKA or PKC & $x_{8}+x_{9}-x_{8} x_{9}$ \\
JNK $=x_{11}$ & PKA or PKC & $x_{8}+x_{9}-x_{8} x_{9}$ \\
\hline
\end{tabular}

These maps are derived from the interaction diagram Figure 2 of [21, where incoming directed nodes are combined by logical disjunction, that is, the or operation. Other dynamics are also compatible with known interactions in this network, and the algebraic method presented here applies to all.

For the two nonconstant steady states, the inclusive and exclusive basins of attraction are the same, and both count 512 states.

A basis for the ideal of the first steady state 111110111111 is given by $\left\{x_{5}, x_{3}-1, x_{11}^{2}-x_{11}, x_{10}^{2}-x_{10}, x_{9}^{2}-x_{9}, x_{8}^{2}-x_{8}, x_{7}^{2}-x_{7}, x_{6}^{2}-x_{6}, x_{4}^{2}-x_{4}, x_{2}^{2}-x_{2}, x_{1}^{2}-x_{1}\right\}$, and one can see a vanishing configuration of $s_{5}=1$. The Hilbert function drops down from $\sum_{i=0}^{t}\left(\begin{array}{c}11 \\ i\end{array}\right)$ at $t^{\star}=1$ consistent with Theorem 3.1. 


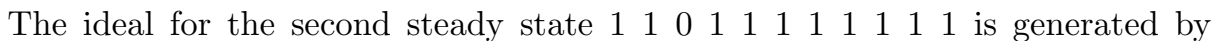
$\left\{x_{5}-1, x_{3}, x_{11}^{2}-x_{11}, x_{10}^{2}-x_{10}, x_{9}^{2}-x_{9}, x_{8}^{2}-x_{8}, x_{7}^{2}-x_{7}, x_{6}^{2}-x_{6}, x_{4}^{2}-x_{4}, x_{2}^{2}-x_{2}, x_{1}^{2}-x_{1}\right\}$ and shows vanishing configuration $s_{5}=0$, again located at size $k=1$ by the Hilbert function.

\section{Conclusions}

Many properties of biological networks with both deterministic and stochastic dynamics can be expressed in the language of commutative algebra, and computations can also be done exactly in this framework with existing algorithms and software. We have illustrated the translation and computations in examples showing that the algebra is computationally feasible on real problems.

\section{REFERENCES}

[1] Réka Albert and Hans G. Othmer, The topology of the regulatory interactions predicts the expression pattern of the segment polarity genes in Drosophila melanogaster, J. Theoret. Biol. 223 (2003), no. 1, 1-18, DOI 10.1016/S0022-5193(03)00035-3. MR2069236

[2] Eric Babson, Shmuel Onn, and Rekha Thomas, The Hilbert zonotope and a polynomial time algorithm for universal Gröbner bases, Adv. in Appl. Math. 30 (2003), no. 3, 529-544, DOI 10.1016/S0196-8858(02)00509-2. MR1973955 (2004h:13030)

[3] Madalena Chaves, Réka Albert, and Eduardo D. Sontag, Robustness and fragility of Boolean models for genetic regulatory networks, J. Theoret. Biol. 235 (2005), no. 3, 431-449, DOI 10.1016/j.jtbi.2005.01.023. MR2158953

[4] David Cox, John Little, and Donal O'Shea, Using algebraic geometry, Graduate Texts in Mathematics, vol. 185, Springer-Verlag, New York, 1998. MR.1639811 (99h:13033)

[5] Ian H. Dinwoodie, Sequential importance sampling of binary sequences, Stat. Comput. 22 (2012), no. 1, 53-63, DOI 10.1007/s11222-010-9205-0. MR2865055

[6] I. H. Dinwoodie, Conditional tests on basins of attraction with finite fields. Methodol. Comput. Appl. Probab. 16 (2014), no. 1, 161-168. MR3169081

[7] W. Decker, G.-M. Greuel, G. Pfister, and H. Schönemann (2011), Singular 3-1-3 — A computer algebra system for polynomial computations. http://www.singular.uni-kl.de.

[8] Franziska Hinkelmann, David Murrugarra, Abdul Salam Jarrah, and Reinhard Laubenbacher, A mathematical framework for agent based models of complex biological networks, Bull. Math. Biol. 73 (2011), no. 7, 1583-1602, DOI 10.1007/s11538-010-9582-8. MR2802438 (2012f:92003)

[9] S. Klamt, J. Saez-Rodriquez, J. A. Lindquist, L. Simeoni, and E. D. Gilles, A methodology for the structural and functional analysis of signalling and regulatory networks, BMC Bioinformatics 7 (2006), 1471-2105.

[10] Martin Kreuzer and Lorenzo Robbiano, Computational commutative algebra. 1, SpringerVerlag, Berlin, 2000. MR,1790326 (2001j:13027)

[11] L. Mendoza, A network model for the control of the differentiation process in Th cells, BioSystems 84 (2006), 101-114.

[12] M. K. Morris, J. Saez-Rodriguez, P. K. Sorger, and D. A. Lauffenburger, Logic-based models for the analysis of cell signaling networks, Biochemistry 49 (2010), 3216-3224.

[13] Giovanni Pistone, Eva Riccomagno, and Henry P. Wynn, Algebraic statistics, Computational commutative algebra in statistics. Monographs on Statistics and Applied Probability, vol. 89, Chapman \& Hall/CRC, Boca Raton, FL, 2001. MR2332740 (2008f:62098)

[14] A. Saadatpour, R-S. Wang, A. Liao, X. Liu, T. P. Loughran, I. Albert, and R. Albert, Dynamical and structural analysis of a T cell survival network identifies novel candidate therapeutic targets for large granula lymphocyte leukemia, PLoS Comp. Biol. 7 (2011), e1002267.

[15] K. Sachs, O. Perez, D. Pe'er, D. A. Lauffenburger, and G. P. Nolan, Causal protein-signaling networks derived from multiparameter single-cell data, Science 308 (2005), 523-529.

[16] Julio Saez-Rodriguez, Luca Simeoni, Jonathan A. Lindquist, Rebecca Hemenway, Ursula Bommhardt, Boerge Arndt, Utz-Uwe Haus, Robert Weismantel, Ernst D. Gilles, Steffen Klamt, and Burkhart Schraven, A logical model provides insights into $T$ cell receptor signaling, PLoS Comput. Biol. 3 (2007), no. 8, 1580-1590, DOI 10.1371/journal.pcbi.0030163. MR2369374 
[17] J. Saez-Rodriquez, L. G. Alexopoulos, M. Zhang, M. Morris, D. A. Lauffenburger, and P. K. Sorger, Comparing signaling networks between normal and transformed hepatocytes using discrete logical models, Cancer Res. 71 (2001), 5400-5411.

[18] R. Schlatter, K. Schmich, I. A. Vizcarra, P. Scheurich, T. Sauter, C. Borner, M. Ederer, I. Merfort, and O. Sawodny, ON/OFF and beyond-A Boolean model of apoptosis. PLoS Comp. Biol. 5 (2009), e1000595.

[19] Brandilyn Stigler, Polynomial dynamical systems in systems biology, Modeling and simulation of biological networks, Proc. Sympos. Appl. Math., vol. 64, Amer. Math. Soc., Providence, RI, 2007, pp. 53-84. MR2359649 (2008i:92019)

[20] R. Thomas, Boolean formalization of genetic control circuits, J. Theor. Biol. 42 (1973), 563-585.

[21] A. V. Werhli, M. Grzegorczyk, and D. Husmeier, Comparative evaluation of reverse engineering gene regulatory networks with relevance networks, graphical gaussian models, and bayesian networks, Bioinformatics 22 (2006), 2523-2531.

[22] R. Zhang, M. V. Shah, J. Yang, S. B. Nyland, X. Liu, J. Yun, R. Albert, and T. P. Loughran, Network model of survival signaling in large granular lymphocyte leukemia, PNAS 105 (2008), 16308-16313.

Department of Mathematical Sciences, Portland State University, Portland, OreGON 97201 